Société d'histoire de la révolution de 1848 et des

révolutions du XIXe siècle

49 | 2014

1814-1815. Expériences de la discontinuité

\title{
Rumeurs et expériences collectives de la discontinuité temporelle (1814-1815)
}

Rumors and collective experiences of discontinuity (1814-1815)

Gerüchte und kollektive Erfahrungen zeitlicher Diskontinuität (1814-1815)

François Ploux

\section{(2) OpenEdition}

Journals

Édition électronique

URL : http://journals.openedition.org/rh19/4742

DOI : $10.4000 /$ rh 19.4742

ISSN : $1777-5329$

Éditeur

La Société de 1848

Édition imprimée

Date de publication : 1 décembre 2014

Pagination : 21-35

ISSN : 1265-1354

Référence électronique

François Ploux, « Rumeurs et expériences collectives de la discontinuité temporelle (1814-1815)»,

Revue d'histoire du XIXe siècle [En ligne], 49 | 2014, mis en ligne le 01 décembre 2014, consulté le 03 mai 2019. URL : http://journals.openedition.org/rh19/4742 ; DOI : 10.4000/rh19.4742 


\section{FRANÇOIS PLOUX}

\section{Rumeurs et expériences collectives de la discontinuité temporelle (1814-1815)}

La prolifération des «fausses nouvelles» dans l'espace public fut l'un des plus curieux symptômes de l'état de fermentation politique que connut la France entre le rétablissement des Bourbons en avril 1814 et la dissolution de la Chambre introuvable le 5 septembre 1816ㄹ. Deux années durant, les canaux habituels de diffusion de l'information furent saturés de rumeurs. À Paris, les propos les plus extravagants circulaient dans les cafés, dans les casernes, dans les salons, en place de Grève, quai de Gesvres, sur les boulevards, etc. Autrement dit partout où s'échangeaient les nouvelles du jour dans l'ordinaire des conversations. Mais aucun département, aucune localité ne semblent avoir été épargnés. Les voyageurs, les marchands ambulants et les soldats démobilisés se chargeaient de propager ces bruits à chaque étape de leur itinéraire. C'est pourquoi les recueils de bulletins de police ou de rapports préfectoraux du début du règne de Louis XVIII ressemblent à des catalogues de bruits "absurdes» sur les affaires du temps. L'ensemble des agents chargés du maintien de l'ordre et de la surveillance de l'opinion pourchassaient sans relâche les colporteurs de fausses nouvelles. Les renseignements collectés étaient transmis aux préfets, qui se chargeaient d'en faire la synthèse. Le ministre de l'Intérieur ou le directeur de la Police du royaume puisaient dans cette correspondance la matière de leurs rapports quotidiens ${ }^{2}$.

1. Il est évidemment très difficile de mesurer les oscillations temporelles d'un phénomène qui se prête mal à la quantification (une opération de ce type supposerait de définir au préalable une unité élémentaire de dénombrement, dont on ne voit pas en quoi elle pourrait consister). Par ailleurs la qualité comme la quantité des données extraites des sources administratives ou policières dépend très étroitement de la nature plus ou moins répressive du pouvoir qui les a produites. Cela étant dit, il ne fait guère de doute que la fréquence des rumeurs se situait, en 1814 et 1815 , à un niveau rarement atteint. La situation empira brusquement au mois de décembre 1815. Et le premier trimestre 1816 fut encore plus agité. La décrue n'intervint qu'au printemps. Sur cette périodisation, qu'il faudrait affiner, voir Bernard Ménager, Les Napoléon du peuple, Paris, Aubier, 1988, p. 15-22 et François Ploux, De bouche à oreille. Naissance et propagation des rumeurs dans la France du XIXe siècle, Paris, Aubier, 2003, p. 127-170.

2. Pour préparer cet article, nous avons notamment eu recours aux rapports du comte Anglès et du comte Beugnot ainsi qu’aux bulletins quotidiens de police conservés dans la sous-série $\mathrm{F}^{7}$ des Archives nationales. Georges Firmin-Didot, Royauté ou empire. La France en 1814 d'après les rapports inédits du comte Anglès, Paris, Fimin-Didot, 1897; Eugène Welvert, Napoléon et la police sous la Première Restauration d'après les rapports du comte Beugnot au roi Louis XVIII, Paris, Roger et Chernoviz, 1913. 
La lutte contre les forces d'opposition était bien évidemment le motif principal de cette traque obsessionnelle de la rumeur. On soupçonnait des fauteurs de désordre d'abuser de la crédulité des «hommes du peuple» en répandant l'alarme. Mais, de manière plutôt contradictoire, la police utilisait aussi les données recueillies par ses "mouches» comme une source d'information crédible sur l'"état des esprits» et la mesure des opinions ${ }^{3}$.

Pour tenter d'endiguer ce déferlement de faux bruits, les préfets du roi mirent sur pied tout un arsenal du démenti dont le XIX siècle français n'offre pas d'équivalent, du moins à notre connaissance ${ }^{4}$. La contre-offensive du pouvoir, essentiellement fondée sur la répression judiciaire, consista également à informer les habitants. Car si l'interprétation la plus répandue désignait les agents de l'opposition comme les principaux responsables de l'agitation régnante, certains observateurs - à l'image du préfet du $\operatorname{Lot}^{5}-$ mettaient en cause l'insuffisance des communications gouvernementales. Faute d'informations, la curiosité du public aurait trouvé à s'assouvir dans des récits absurdes ou fabuleux. Dans une note de la fin du mois de mai 1814, le comte Anglès évoquait quant à lui une sorte de réaction thermodynamique de l'esprit public : comprimé sous le gouvernement despotique de Napoléon, il se serait brusquement dilaté et menacerait de faire explosion ${ }^{6}$. En août de la même année, l'auteur d'un rapport sur la situation en Lorraine voyait dans la rumeur la conséquence, ou le symptôme, d'une "émancipation presque générale des esprits»: "L'autorité souveraine est dépouillée, [aux yeux du peuple], des illusions et du respect qui la mettaient autrefois à l'abri de toute irrévérence et de toute discussion ${ }^{7}$. La rumeur, était-il ici suggéré - c'est-àdire l'échange de nouvelles dans le cadre de la sociabilité habituelle - aurait ainsi constitué une modalité de l'appropriation du politique par ceux qui en étaient officiellement exclus.

Rien ne pouvait davantage irriter le pouvoir que cette intrusion intempestive du "commun" dans le domaine réservé de la politique. Les autorités étaient en outre très conscientes de ce que la propagation des rumeurs contrariait le travail de refondation de sa légitimité qu’avait engagé la monarchie bourbonienne. On sait que le façonnement de la mémoire collective fut l'un des principaux enjeux de cette entreprise $e^{8}$. L'impératif de réconciliation des deux France, la recherche d'une stabilisation politique, le souci d'éviter toute

3. Pierre Karila-Cohen, L'État des esprits. L’invention de l'enquête politique en France (1814-1848), Rennes, Presses universitaires de Rennes, 2008.

4. Sur ce point, $c f$. François Ploux, De bouche à oreille, op. cit., p. 46-54.

5. «La curiosité [qui résulte de l'ignorance dans laquelle on laisse les habitants concernant la situation du royaume] ne pouvant trouver d'aliments dans les communications que la gouvernement ne lui fait pas, se repaitt avidement et sans examen, des récits fabuleux, des absurdités grossières dont la malveillance a soin de l'entretenir ». Le préfet du Lot au ministre secrétaire d'État à la police générale, 9 novembre $1815, \mathrm{~F}^{7} 9105$.

6. Georges Firmin-Didot, op. cit., p. 17 (bull. des 27-28 mai 1814).

7. Ibidem, p. 84-85 (bull. du 5 août 1814).

8. Sheryl Kroen, Politics and Theater. The Crisis of Legitimacy in Restoration France. 1815-1830, Berkeley, University of California Press, 2000; Natalie Scholz, 'Past and Pathos. Symbolic Practices 
confrontation directe avec le passé proche, déterminèrent cette stratégie de l'oubli, qui trouva sa première formulation dans l'article 11 de la Charte et sa promesse d'amnistie? . Sous l'impulsion du roi, le régime s'efforça d'escamoter le souvenir des institutions politiques qui auraient pu faire figure d'alternatives possibles à la monarchie restaurée. Il s'agissait d'installer symboliquement cette monarchie dans la durée. D'anéantir toute aspiration à un ordre institutionnel concurrent. Et de désigner le règne des Bourbons comme seul horizon raisonnable et légitime ${ }^{10}$.

Or, ce que les rumeurs de 1814 et 1815 mettent en évidence, aussi bien dans les modalités de leur engendrement que dans leurs énoncés, c'est une expérience collective du temps totalement incompatible avec cette représentation d'une histoire définitivement stabilisée. Les Français, d'abord, éprouvèrent de manière particulièrement intense, et sans doute traumatisante, le sentiment de l'instabilité des institutions politiques. Ils vécurent de ce fait dans l'incertitude ${ }^{11}$. Une incertitude propice à la rumeur, et à la manipulation par la rumeur ${ }^{12}$. Mais - deuxième point - les rumeurs du commencement des deux Restaurations furent souvent, en elles-mêmes, des anticipations. Des anticipations implicites, lorsque, à défaut de se présenter sous les espèces d'une prédiction, elles suggéraient l'imminence d'une grave perturbation politique, et contribuaient de ce fait à entretenir ce climat d'expectative caractéristique de la période. Par ailleurs certains bruits annonçaient sans détour un événement sur le point d'advenir. On pense ici, bien sûr, aux rumeurs concernant le retour de Napoléon, de loin les plus nombreuses. On voudrait, dans cet article, interroger le type de rapport au temps que révèle cette gigantesque fausse nouvelle aux allures de prophétie. Mais il nous faudra auparavant dégager quelques propriétés du régime d'incertitude qui, de toute évidence, en a favorisé l'éclosion.

of Reconciliation during the French Restoration', History and Memory, Vol. 22, n ${ }^{\circ}$ 1, Spring/Summer 2010, p. 48-79.

9. Bettina Frederking, " "Il ne faut pas être le roi de deux Peuples»: Strategies of National Reconciliation in Restoration France', French History, 22, 4, déc. 2008, p. 446-468.

10. Les transferts de souveraineté s'accompagnèrent, en 1814 et 1815, de flambées iconoclastes. Voir sur ce point les travaux d'Emmanuel Fureix, notamment "L'iconoclasme politique au XIXe siècle», in Annie Duprat [dir.], Révolutions et mythes identitaires. Mots, violences, mémoire, Paris, Nouveau Monde éditions, 2009, p. 173-194 et «L'iconoclasme : une pratique politique? (1814-1848)», dans Laurent Le Gall, Michel Offerlé, François Ploux [dir.], La politique sans en avoir l'air. Aspects de la politique informelle. XIX'-XXI siècle, Rennes, Presses universitaires de Rennes, 2012, p. 117-131.

11. "L'opinion publique est, dit-on, toujours au même degré d'incertitude et est toujours tourmentée par les malveillants». Bull. du 31 octobre 1814, Arch. nat. $\mathrm{F}^{7} 3837$. C'est le type de phrase dont les bulletins de police regorgent.

12. Pierre Bourdieu, Méditations pascaliennes, Paris, Seuil, «Liber», 1997, p. 270. 


\section{QuAND RÈGNE L'INCERTITUdE}

Dans un rapport du 22 novembre 1814, le comte Beugnot signalait au roi l'une des innombrables rumeurs dont ses agents le tenaient informé au jour le jour : "On répand dans Paris qu'il y a encore eu l'avant-dernière nuit une fausse alerte aux Tuileries. [...] On aurait peine à se figurer quels effets ces bruits, en s'enflammant le lendemain dans la capitale, y produisent. Toutes les têtes se remplissent de projets de conspirations, de machinations contre le gouvernement, d'incertitudes sur le présent et de dangers pour l'avenir. De Paris, ces fables courent les départements et y exercent encore plus de ravages parce qu'il est plus difficile de les y détruire $»^{13}$. Ces quelques phrases, outre qu'elles évoquent l'existence d'un mécanisme circulaire d'engendrement réciproque de la rumeur et du sentiment d'incertitude, suggèrent que cette incertitude plongea les Français dans un état d'expectative. Ä Paris comme en province, on vécut dans l'attente d'un événement. Une situation propice à la prolifération de rumeurs où s'exprimaient, selon les cas, des craintes ou des aspirations, elles-mêmes nourries d'une mémoire collective. Tâchons donc de démêler l'écheveau des facteurs qui ont pu contribuer à l'instauration de ce régime d'incertitude dans lequel la France a basculé à la chute de l'empire.

\section{L'instabilité du présent}

Il faut d'abord attribuer au sentiment largement répandu d'une extrême vulnérabilité du pouvoir l'anxiété chronique dont rendaient compte les fonctionnaires chargés de la surveillance de l'opinion. Une menace permanente semblait planer sur les autorités établies. Cette impression diffuse de l'instabilité des institutions politiques alimentait une certaine conscience du temps. Ce qui allait advenir apparaissait douteux, et faisait l'objet de mille conjectures (un mot repris sans cesse dans les rapports sur l'esprit public). De là une extrême vigilance, génératrice de rumeurs. Le moindre indice semblait signaler une grave perturbation. Deux exemples parmi cent : en décembre 1814, une échauffourée entre douaniers fit croire, dans la Meuse, à une invasion ${ }^{14}$; quelques mois plus tard, le maréchal Ney, étant souffrant, ne put accompagner le comte d'Artois lors d'une apparition publique : le bruit de son arrestation se mit aussitôt à circuler ${ }^{15}$.

Au commencement de l'été 1814, soit trois mois après l'abdication de Fontainebleau, les Parisiens semblaient convaincus de l'imminence d'un changement. Il n'était question, dans les conversations dont les archives

13. Eugène Welvert, op. cit., p. 277 (bull. du 22 nov. 1814).

14. Bull. du 15 déc. 1814 , Arch. nat. $\mathrm{F}^{7} 3733$.

15. Bull. du 15 novembre 1814, Arch. nat. F7 3837. Le bulletin de police du 7 juillet 1814 mettait en garde contre l'usage, dans un projet de loi sur le Conseil d'État, de l'expression «Conseil d'en haut ": "Dans un moment où l'opinion, trop souvent incertaine, s'attache à tout épier, à tout eommenter [raturé dans l'original], à tirer de tout des inductions plus ou moins hasardées, cette expression a dû paraître ature draturé dans l'original] ». Arch. nat. F7 3836. 
portent les traces, que du prochain renversement des Bourbons. "On dit [...] dans les promenades, dans tous les lieux publics, dans les maisons particulières, que l'ordre actuel des choses ne peut subsister longtemps, qu'une tempête se prépare. [...] On dit encore que le comte d'Artois, qui est malade de chagrin, répète souvent nous ne pourrons pas tenir" (bull. du 16 juillet 1814$)^{16}$. La police rapportait tous les jours des propos de ce genre : les Bourbons «ne pourront se soutenir» (bull. du 19 juillet); «Le gouvernement ne continuera pas longtemps" (bull. du 20 juillet 1814); "Avant trois mois les Bourbons seront renversés» (bull. du 20 juillet); «Les Bourbons se soutiendront quelque temps, mais pas longtemps» (bull. du 21 juillet); "Louis XVIII ne sera pas longtemps sur le trône» (bull. du 23 juillet), etc.

On disait les Bourbons impopulaires. C'est pourquoi l'on s'attendait à ce qu'ils soient renversés. En août, on répétait partout qu'à moins que la «situation du Royaume» ne s'améliore très vite, il fallait craindre "une catastrophe ${ }^{17}$. On annonçait encore du "mic-mac», une "tempête», du "tapage ${ }^{18}$. Autrement dit une insurrection ou un coup d'État militaire ${ }^{19}$. On disait début septembre que l'influence grandissante des jésuites et des royalistes exagérés finirait par provoquer un soulèvement "général» contre les Bourbons ${ }^{20}$. "Il est impossible que cela dure», affirmait-on au même moment dans une maison bonapartiste ${ }^{21}$. "Ils ont beau faire, déclarait un colonel, ils ne tiendront pas $»^{22}$. À partir du 5 octobre, les bulletins constataient, en même temps qu'une augmentation du prix du pain, une détérioration de l'esprit public. Les propos alarmants se multipliaient, y compris dans les milieux proches du pouvoir : on parlait d'un «mouvement", d'un soulèvement au sujet des grains, de la proclamation de la République ${ }^{23}$, etc.

On peut raisonnablement faire l'hypothèse que cette croyance dans l'imminence d'un changement, parce qu'elle supposait une aptitude à concevoir la possibilité d'une alternative politique, découlait directement de l'expérience encore fraîche de bouleversements en série dans la sphère du pouvoir. Thomas Kselmann met en relation la culture prophétique qui, selon lui, caractérisa les premières années de la Restauration avec une crise de la légitimité politique ${ }^{24}$. Natalie Scholz observe de son côté que la confrontation des répertoires symboliques a pu donner de la visibilité à l'instabilité poli-

16. Bull. du 16 juillet 1814, Arch. nat. $\mathrm{F}^{7} 3836$.

17. Bull. du 10 août 1814, Arch. nat. $\mathrm{F}^{7} 3836$.

18. Bull. du 14 juillet, 18 août, 23 août, $1^{\text {er }}$ septembre 1814, Arch. nat. $F^{7}$ 3836. La tension semble être montée d'un cran à l'approche de la Saint-Louis. Bull. 3 août 1814, Arch. nat. F7 3836.

19. Le bulletin du 14 juillet 1814 notait que les officiers résidant à Paris "comptent sur un prochain mouvement». Arch. nat. $\mathrm{F}^{7} 3837$.

20. Bull. du $1^{\text {er }}$ septembre 1814, Arch. nat. $\mathrm{F}^{7} 3836$.

21. Bull. du 6 septembre 1814, Arch. nat. F7 3836.

22. Bull. du 26 septembre 1814, Arch. nat. F7 3836.

23. Bull. des 10, 15, 17 et 18 octobre 1814, Arch. nat. F7 3837.

24. Thomas A. Kselman, Miracles and Prophecies in Nineteenth-Century France, New Brunswick, New Jersey, Rutgers University Press, 1983, 283 p. 
tique $^{25}$. Partisans et adversaires des Bourbons se livrèrent, y compris dans les campagnes, à une véritable guerre des emblèmes ${ }^{26}$. Pareille mise en scène de l'affrontement des légitimités contribuait à rendre sinon plausible, du moins concevable, l'établissement d'un ordre politique concurrent.

La circulation des rumeurs entretenait ce sentiment du précaire. Les Français vivaient dans l'attente d'un bouleversement majeur. En province, des attroupements se formaient aux relais de poste. Au Puy, où, d'après un rapport du 22 avril 1815, les royalistes cherchaient à alarmer la population en répandant des fausses nouvelles, "les courriers sont attendus avec impatience comme devant annoncer de grands événements ${ }^{27}$. Trois jours plus tard, le bruit s'étant propagé à Tarbes que l'empereur avait été assassiné, cinq cents personnes s'assemblèrent sur la place en attendant la malle-poste de Paris ${ }^{28}$.

\section{La manipulation}

Les rumeurs annonciatrices d'un ébranlement politique, à défaut d'être nécessairement séditieuses, n’en présentaient pas moins un réel danger pour le pouvoir, potentiellement fragilisé par la mise en question de sa stabilité. C'est pourquoi la circulation de ces bruits en 1814 et 1815 fait immédiatement surgir l'hypothèse d'une machination. À n'en pas douter, la rumeur occupait une place de choix dans la gamme des procédés au moyen desquels certains opposants s'efforçaient d'ébranler le pouvoir ${ }^{29}$. Il s'agissait notamment de persuader les habitants que le régime en place était condamné à brève échéance. Contentons-nous, pour faire court, d'évoquer à ce propos les menées des royalistes durant les Cent-Jours. Des nobles ${ }^{30}$ et des prêtres ${ }^{31}$ restés fidèles aux Bourbons, en même temps qu'ils diffusaient clandestinement des brochures de propagande - et parfois des proclamations apocryphes ${ }^{32}-$ répandaient de fausses informations destinées à semer l'inquiétude dans les esprits, à ralentir les mouvements de ralliement en faveur de Napoléon, et à encourager l'insoumission. Le clergé du Doubs allait répétant que Napoléon ne faisait que passer ${ }^{33}$. Ailleurs on annonçait qu'il était mort, que l'Empire

25. Natalie Scholz, 'Past and Pathos', loc. cit.

26. Cf. Emmanuel Fureix, loc. cit.

27. Bull. du 29 avril 1815, F7 3734.

28. Bull. du 10 mai 1815, Arch. nat. F7 3734. Une scène identique eut lieu au Havre le 6 mai (Bull. du 8 mai 1815, F7 3734).

29. Il n'est pas impossible que cet usage subversif de la rumeur se soit constitué sous le règne de Napoléon, dans un contexte de rétractation de l'activité politique dans l'occulte. La Restauration aurait hérité de pratiques expérimentées dans les dernières années du règne, semble-t-il avec un certain succès. Cf. Natalie Petiteau, Les Français et l'Empire (1799-1815), Paris, Boutique de l'Histoire, 2008.

30. Bull. du $1^{\text {er }}$ mai 1815, Arch. nat. $\mathrm{F}^{7} 3734$. À en croire un bulletin daté de Lyon, les nobles de la ville utilisaient la rumeur non seulement pour semer l'inquiétude dans la population, mais encore pour entrer en relation avec «l'homme du peuple».

31. Voir, dans le carton $\mathrm{F}^{7} 3734$, l'ensemble des bulletins correspondant à la période des CentJours. Émile Le Gallo, Les Cent-Jours. Essai sur l'histoire intérieure de la France depuis le retour de l'âle d'Elbe jusqu'à la nouvelle de Waterloo, Paris, Alcan 1924, p. 335, 341, 349, 352, 361.

32. Telle la fausse proclamation de Francfort. Émile Le Gallo, op. cit., p. 331.

33. Ibidem, p., 352. 
touchait à sa fin, ou que le roi s'apprêtait à remonter sur le trône ${ }^{34}$. Et l'on sait que les fédérations de 1815 s'étaient donné pour tâche d'épauler les autorités dans leur effort de neutralisation des rumeurs ${ }^{35}$. La Chambre des représentants se saisit même du problème. D'abord, le 17 juin 1815, dans le cadre d'une proposition de loi sur la répression des provocations séditieuses et des abus de la presse. Le député Jacques de Maleville, dans sa présentation du texte, eut cette remarque lucide : "Comment concilier la confiance qui est due au gouvernement [...] avec la menace de le renverser, avec l'annonce qu'il sera renversé, changé ou détruit? Contester au gouvernement sa stabilité, sa légitimité, c'est fournir des armes à ses ennemis, c'est le dépouiller de sa puissance, de celle de l'opinion publique ${ }^{36}$.» À compter du 25 juin, les deux Chambres examinèrent un projet de loi de sûreté publique rédigé par la Commission exécutive que présidait Fouché. L'une des principales dispositions de ce texte concernait la publication de fausses nouvelles au moyen de placards, d'imprimés ou "par des discours tenus dans les lieux ou réunions publiques». Des voix s'élevèrent pour dénoncer une loi autorisant la police à arrêter un citoyen ayant en toute innocence colporté une information erronée ${ }^{37}$.

\section{Une défiance généralisée}

Dans un contexte de durcissement des antagonismes politiques, et alors que planait partout la menace de règlements de comptes, nombreux étaient ceux qui s'inquiétaient des intentions du parti adverse. La défiance fut une autre dimension de ce régime d'incertitude que mettent en évidence les sources policières ${ }^{38}$. Ainsi pourrait-on mentionner toute une série de rumeurs d'agressions qui se propagèrent là où deux communautés rivales se trouvaient en situation d'opposition frontale. Dans la Loire-Inférieure et les Deux-Sèvres, les bruits - faux - de mobilisation d'acquéreurs de biens nationaux, de Polonais de la Garde, de douaniers, de partisans de Napoléon, déclenchèrent au début du mois de mai 1814 plusieurs paniques spectaculaires $^{39}$. Dans le Gard de la Terreur blanche, la rumeur alimenta l'animosité entre partisans et adversaires des Bourbons ${ }^{40}$. Auparavant, sous la première

34. Ibidem, p. 329-30, 342.

35. Robert S. Alexander, Bonapartism and Revolutionary Tradition in France. The Fédérés of 1815, Cambridge, Cambridge University Press, 1991.

36. Archives parlementaires de 1787 à 1860. Recueil complet des débats législatifs et politiques des Chambres françaises, $2^{\mathrm{e}}$ série, T. XIV, Paris, Librairie administrative de Paul Dupont, 1869, Archives parlementaires, séance du 17 juin 1815 (Chambre des représentants), p. 473.

37. Ibidem, p. 533 et suiv. Voir les interventions de Boissy d'Anglas (p. 534-535), du comte de Ségur (p. 545), du comte Dedeley d'Agier (p. 545).

38. On laissera de côté ici la question, cependant essentielle à la compréhension des mécanismes de la rumeur, du peu de crédit accordé aux informations «officielles».

39. Arch. nat. F7 3733 et Aurélien Lignereux, «La Première Restauration face à l'“inexplicable Vendée" : la levée de boucliers des 3-5 mai 1814", Annales de Bretagne et des pays de l'Ouest, tome 118, 4, 2011, p. 63-89.

40. Pierre Triomphe, «Des bruits qui courent aux mots qui tuent. Rumeurs et violences dans le Gard en 1815", Revue d'histoire du XIX'e siècle, n 36, 2008/1, p. 59-73. 
Restauration, plusieurs rumeurs de "double conspiration" avaient provoqué une grande agitation dans la capitale. Les royalistes "zélés» voyaient des complots partout. Mais les notables compromis pour avoir servi Napoléon ou siégé dans les assemblées révolutionnaires craignaient également pour leur vie (ils s'affolèrent à l'approche du 21 janvier 181541). L'automne 1814 fut particulièrement agité. Une grande fermentation régnait dans Paris ${ }^{42}$. Fin novembre, le bruit d'un coup de force des officiers bonapartistes contre le gouvernement se répandit en même temps que la rumeur d'un attentat des anciens chefs vendéens contre les généraux de Napoléon ${ }^{43}$. Tandis que les militaires se terraient, on prenait des précautions extraordinaires aux Tuileries. "Crainte, défiance, alarmes ont circulé d'un parti à l'autre " ${ }^{44}$. Et elles se propagèrent depuis les élites proches du pouvoir vers le peuple (il fallut organiser des patrouilles nocturnes pour rassurer les Parisiens ${ }^{45}$ ) et de Paris vers la province.

Il ne fait aucun doute que l'anarchie policière caractéristique de la première Restauration, associée à l'obsession du complot dans l'entourage du comte d'Artois ${ }^{46}$, ont beaucoup contribué à instaurer ce climat d'inquiétude, de suspicion et de rumeur ${ }^{47}$. Beugnot s'en prenait directement aux royalistes exagérés : «Le mal qu'ils produisent est immense, parce que, à force de feindre des dangers, ils les feront naître en inquiétant le peuple et en lui montrant l'État comme en péril continuel ${ }^{48}$." Le ministre ne pouvait que constater les effets désastreux du système de surveillance des lieux publics par les mouches de la police - étaient notamment mises en cause les polices parallèles du pavillon de Marsan. Ces informateurs, pour tester les réactions des ouvriers qu'ils étaient chargés d'espionner, répandaient des bruits au sujet de Napoléon. Et lorsque la rumeur - qu'ils avaient eux-mêmes propagée - du retour de l'empereur leur était rapportée, ils se dépêchaient d'en informer les Tuileries, où on les récompensait pour ce service ${ }^{49}$. On est en présence, ici,

41. Georges Firmin-Didot, op. cit., p. 191-194 et 223-226 (bull. du $1^{\text {er }}$ et du 19 janvier 1815). La célébration de la mort de Louis XVI a servi de support à une entreprise d'expiation collective, comportant un versant pénitentiel et une dimension sacrificielle. Les rumeurs qui circulèrent à Paris à l'approche de la commémoration du régicide témoignent de l'intériorisation de ce discours par les opposants. Selon une première rumeur, les conventionnels devaient gagner Saint-Denis pieds nus, une torche au poing et la corde au cou. Une autre rumeur annonçait une Saint-Barthélemy des républicains. Emmanuel Fureix, La France des larmes. Deuils politiques à l'âge romantique (1814-1840), Seyssel, Champ Vallon, 2009, p. 178-179.

42. Les bulletins de police enregistrèrent une anxiété croissante. Bull. des 5, 8, 12 et 14 octobre 1814, Arch. nat. $\mathrm{F}^{7} 3837$, et tous ceux qui suivent.

43. Bull. du 24 novembre 1814, Arch. nat. F7 3837.

44. Eugène Welvert, op. cit., p. 285-286 (bull. du 26 nov. 1814).

45. Idem.

46. Eugène Welvert, op. cit., p. 30 et sq. (Bull. des 30 juin et $1^{\text {er }}$ juill. 1814).

47. Nous avons développé ce point dans : "Le fantôme de Napoléon. Histoire d'une rumeur", in Stéphanie Sauget [dir.], Les âmes errantes. Fantômes et revenants, Grâne, Creaphis, 2012, p. 17-35.

48. Eugène Welvert, op. cit., p. 294 (bull. du 30 nov. 1814).

49. Ibidem, p. 11-12 et 16-18 (bull. du 17 et du 18 juin 1814). Le bulletin du 4 novembre 1814 mentionne le témoignage d'un jeune garde du corps qui tend à confirmer ce système d'espionnage à base de bruits provocateurs. 
d'une prédiction créatrice : le pavillon de Marsan croyait en l'existence d'un complot bonapartiste; il faisait surveiller les ouvriers et les militaires par des espions qui, pour tester les réactions de ces éléments suspects, répandaient que Napoléon allait bientôt les délivrer. Ce à quoi les ouvriers finirent par croire. «À force de supposer des dangers, écrivait Jules Anglès, alors directeur général de la Police, on finira par les faire naître, en frappant continuellement les esprits d'un retour impossible à tenter dans l'état actuel de l'Europe ${ }^{50}$."

\section{La colère et la peur}

«L'esprit public ne paraît pas s'améliorer, constatait l'auteur du bulletin du 6 juillet 1814. Dans la classe du peuple et parmi les militaires, toujours mêmes regrets du passé, même mécontentement du présent, mêmes idées pour l'avenir ${ }^{51}$." Dans les mois qui suivirent les deux restaurations monarchiques, le ressentiment à l'égard du régime et de ses soutiens entretint la nostalgie d'un empire qu'on semblait pourtant n'avoir, dans un premier temps, que peu regretté. Mais ce "mécontentement du présent " qu'évoquait le document cité, mélange de rancour, de peur et de colère, explique aussi l'état d'expectative dans lequel baignaient les Français. Pas seulement parce que chacun pouvait constater l'impopularité du régime, et prendre la mesure de sa fragilité. Dans ce contexte trouble, instable, anxiogène, l'insatisfaction du plus grand nombre, faute de pouvoir déboucher sur la formulation d'un ensemble cohérent de revendications - quinze années de dictature consulaire et impériale ayant détruit toute espèce de médiations dans l'espace public - engendrait des rumeurs anticipatrices où s'exprimaient peurs et aspirations collectives.

Il n'est pas inutile de rappeler que la chute de Napoléon avait fait naître l'espoir d'une exemption fiscale. Les promesses royalistes d'une abolition des droits réunis avaient produit une attente, très vite déçue. Pour beaucoup de Français, en particulier dans les campagnes, l'avènement de Louis XVIII coïncida avec une immense déception ${ }^{52}$. D'autres sujets de mécontentement n'allaient pas tarder à surgir, qu'il est inutile d'inventorier ici tant ils sont connus. En outre, il n'est pas impossible que l'ouverture du congrès de Vienne ait provoqué un surcroît d'inquiétude. Elle coïncide en effet avec une nette détérioration de l'esprit public. On ne parle à Paris, était-il écrit dans le bulletin du 15 novembre 1814, que d'assassinats, du mécontentement des militaires, de l'influence des prêtres, des prétentions des émigrés, du mauvais esprit des départements. «On dit particulièrement, et plusieurs rapports s'accordent en ce point, que l'on doit nous enlever, par suite des opérations du congrès de Vienne, la Franche Comté et une partie de la Lorraine et de l'Alsace [...]. Ces bruits

50. Georges Firmin-Didot, Royauté ou empire..., op. cit., bull. du 23 juillet 1814.

51. Bull. du 6 juillet 1814 , Arch. nat. F7 3836.

52. L'histoire des troubles antifiscaux de 1814-1815 reste à faire. 
désastreux, en portant l'inquiétude dans tous les esprits, fournissent aux malveillants l'occasion de desservir le gouvernement de Sa Majesté et de ranimer les partis ${ }^{53} . "$

Mais il faut surtout insister sur l'impact des controverses relatives à la restitution des propriétés nationales. Les "acquéreurs " ${ }^{54}$ commencèrent à s'inquiéter dès la fin du mois d'avril 1814. L'examen, au cours de l'automne, du projet de loi sur la rétrocession des biens non vendus n'arrangea pas les choses. Au même moment, les incidents se multipliaient en province (on signalait des reprises de possession, des menaces, des pressions morales, etc.) Un vent de panique s'empara de centaines de milliers de Français. Et la peur ne tarda pas à gagner les strates inférieures de la société rurale, car il était aussi question du rétablissement des rentes féodales ${ }^{55}$. On saisit mieux, ainsi, comment a pu germer l'espoir d'une alternative politique qui aurait mis fin à ces menaces.

\section{LES AVATARS D'UNE PROPHÉTIE}

\section{Une attente collective}

Les fausses nouvelles concernant Napoléon ne semblent pas avoir commencé à circuler immédiatement après chacune des deux abdications. En mai 1814 et en juillet 1815, il fut peu question du souverain déchu dans les conversation ${ }^{56}$. Mais, très vite, les rumeurs napoléoniennes émergèrent dans le tourbillon des bruits sinistres qui obsédaient les habitants. Comme si, dans une logique en quelque sorte compensatoire, la peur avait engendré, du moins chez certains, l'espérance ${ }^{57}$.

Au commencement du printemps 1814, deux questions préoccupaient la police : l'agitation antifiscale et les menaces dont étaient ou se croyaient victimes les propriétaires de biens nationaux. Mais à la fin du mois de mai, les autorités constataient qu'on parlait beaucoup de Napoléon dans Paris. C'est ce que notait le bulletin des 25 et 26 mai : «Cafés, lieux publics - On s'entretient sur le sort de Bonaparte à l'île d'Elbe. L'autorité dont il jouit donne lieu à beaucoup de commentaires. On se demande si les puissances n'ont pas une arrière-pensée en lui conservant une existence politique ${ }^{58}$.» En

53. Bull. du 15 novembre 1814 , Arch. nat. $\mathrm{F}^{7} 3837$.

54. Une catégorie qui n'est jamais désignée, dans les sources, que sous cette forme générique et à tout prendre très vague.

55. Certains bulletins de police accusaient les acquéreurs d'avoir délibérément cherché à effrayer le petit peuple en agitant la question des droits seigneuriaux. Bull. du 6 déc. et du 28 déc. 1814, F7 3633.

56. Seule une recherche approfondie dans une documentation foisonnante pourrait confirmer cette première impression.

57. On se souvient que Georges Lefebvre avait fait de l'inquiétude et de l'espérance deux caractères affectifs de la «mentalité révolutionnaire». "Les foules révolutionnaires», article repris dans La Grande Peur de 1789, Paris, Colin, 1988, p. 257-259.

58. Eugène Welvert, op. cit., p. 4-5 (bull. des 25 et 26 mai 1814). 
juin, il n'était question, place de Grève, quai de Gesvres, dans les salons du faubourg Saint-Germain, dans les casernes, sur les boulevards, dans les cafés, que de l'ex-empereur ${ }^{59}$. Le bulletin du 20 juillet signalait que «les ouvriers [des faubourgs Saint-Antoine et Saint-Marceau] sont violemment animés contre le gouvernement. Ils parlent sans cesse de Bonaparte qui, disent-ils, ne soutenait pas les prêtres ${ }^{60}$. Les militaires, qu'on voyait se mêler aux ouvriers assemblés quai de Gesvres ou devant l'Hôtel de Ville, étaient parfois désignés comme responsables de la dégradation de l'esprit public ${ }^{61}$. En province, le bruit du retour de Napoléon circulait dès le mois de juin ${ }^{62}$. Au début du mois d'août, dans les Ardennes, et plus particulièrement à Mézières et Charleville, on ne parlait "que de l'ex-empereur ${ }^{63}$ ». Jusqu'à l'annonce du débarquement au golfe Juan, la rumeur n'allait cesser d'enfler.

La seconde abdication inaugura un scenario à peu près identique. En juillet 1815, l'opinion s'inquiétait surtout du démantèlement de la France par les coalisés. On s'entretenait également d'un mouvement insurrectionnel à Paris, de l'abdication du roi, etc. Mais, dès le mois d'août, le nom de l'exempereur était dans toutes les conversations. La rumeur de son retour, qui allait bientôt prendre des proportions inouïes, se répandait alors même que continuaient de circuler des bruits anxiogènes de révolte, de massacres, de rétablissement des servitudes féodales, d'annulation des ventes nationales... Le bruit public mêlait constamment ces deux registres. L'attente du retour de celui que beaucoup assimilaient à un sauveur ne peut s'expliquer que par la crainte tenace d'une réaction aristocratique. Les rumeurs désignaient l'avenir proche simultanément comme une menace et comme une délivrance ${ }^{64}$. Toutefois, en 1814 comme en 1815, le thème du retour de Napoléon fut l'élément prépondérant dans le bouillonnement des rumeurs ${ }^{65}$. Au grand scandale du pouvoir, qui aurait voulu qu'on oublie ce personnage encombrant («Pourquoi parler sans cesse de lui quand il ne s'agit que de le faire oublier? $\left.{ }^{66} »\right)$.

59. Eugène Welvert, op. cit., p. 8 sq. (bull. des 13, 17-18, 22, 29 juin, etc.)

60. Bull. du 20 juillet 1814, Arch. nat. F7 3836.

61. Bull. des 6, 16 et 19 août 1814, Arch. nat. F7 3836. Dans les marchés et dans le faubourg Saint-Antoine, on disait que le roi faisait passer des charrettes de blé en Angleterre.

62. Bull. des 16 et 20 juin 1814, Arch. nat. F7 3733.

63. Bull. du 11 août 1814, Arch. nat. F7 3836.

64. Certains bulletins mettaient très clairement en évidence ce mélange - potentiellement détonnant - de colère, d'angoisse et d'espérance. En Alsace, par exemple, les paysans se plaignaient de la levée des contributions. La crainte du rétablissement des dîmes et des rentes les tenait dans l'agitation. Et ils répétaient que Napoléon s'avançait à la tête d'une armée de Turcs. (Bull. du 26 août 1815, Arch. nat. $\mathrm{F}^{7}$ 3733). L'épisode, postérieur, des troubles frumentaires de 1816 se caractérise également par la superposition de rumeurs dénonciatrices et de rumeurs quasi messianiques (François Ploux, De bouche à oreille, op. cit., p. 160-170)

65. Pour un instantané en novembre 1815, voir l'ensemble des rapports du carton Arch. nat. $\mathrm{F}^{7} 3823$

66. Bull. du 9 juillet 1814, Arch. nat. $\mathrm{F}^{7} 3836$. Et, le 13 septembre : «On croit qu'il serait d’une très grande importance de donner aux journalistes l'ordre de ne plus parler de Bonaparte dans leurs feuilles». (Arch. nat. F7 3836). 
La politique d'amnésie sélective de la monarchie se heurta à cette parole incontrôlable. Pour un nombre incalculable de Français - parisiens ou provinciaux, citadins ou villageois -, l'Empire semblait une alternative crédible, et souhaitée ${ }^{67}$, à la monarchie restaurée. La rumeur napoléonienne fut le symptôme d'une formidable attente collective. Mais il faut s'interroger sur le type de rapport à l'avenir que pouvaient recouvrir ces nouvelles prospectives. Pour tenter d'y voir plus clair, il peut être utile d'établir une typologie des formes d'énonciation de la rumeur. Tout en étant bien conscient de ce que les catégories proposées sont forcément un peu arbitraires.

\section{Les formes de la croyance}

Bien souvent, la fausse nouvelle, dont il faut observer qu'elle était fréquemment agrémentée de propos hostiles à l'égard du monarque, n'était rien d'autre qu'une provocation (mal) déguisée, qu'un défi lancé au pouvoir, qu'une manière détournée de contester la légitimité des Bourbons ${ }^{68}$. Ou d'exprimer, sous une forme vaguement politique, un mécontentement que le maintien des droits réunis, l'augmentation du prix des denrées, l'influence grandissante du clergé, les mises en cause réelles ou supposées des acquis de la Révolution ne cessaient d'alimenter. L'extraordinaire emprise de la rumeur en 1814-1816 serait alors à mettre en relation avec la neutralisation, sous le Consulat et l'Empire, de l'ensemble des dispositifs susceptibles de médiatiser, dans l'espace public, le ressentiment et les aspirations populaires. Et de les retraduire dans le langage de la politique. En sa forme séditieuse, la rumeur aurait été l'expression spontanée d'une conscience politique fruste, inorganisée.

Elle conservait ce caractère essentiellement provocateur et contestataire lorsqu'elle empruntait le canal de l'écrit, ou lorsqu'elle circulait sous la forme matérialisée d'objets - cartes ${ }^{69}$, médailles $^{70}$ ou fausses pièces ${ }^{71}$ - représentant l'empereur. On pourrait encore mentionner quantité d'airs ou de chansons sur le thème du retour de Napoléon. L'intention séditieuse était ici sans équivoque : il s'agissait d'anéantir les efforts déployés par le pouvoir pour asseoir la légitimité de la monarchie en l'inscrivant dans la durée historique. En

67. Pas chez tous ni partout, il est vrai. Il faudrait procéder à une étude plus fine de la circulation de ces bruits dans les contrées réfractaires (Ouest ou Midi blancs).

68. Le 3 novembre 1815 , un manouvrier et un serrurier attablés dans un cabaret de Montieren-Der [Haute-Marne] se répandent en propos séditieux après qu'un autre client a crié Vive le Roi : "Gros cochon, vache, roi Cotillon, Vive l'Empereur, merde aux Bourbons! Dans un mois Napoléon reviendra à la tête d'une grande armée, chassera les Bourbons et fera étrangler tous les royalistes ". Pierre G. Jacquot, Opposition et terreur blanche en Haute-Marne sous la Restauration ou la fidélité aux souvenirs, Saint-Dizier, Imp. Jean Gauthier, 1981, p. 52-53.

69. Répandues dans les couloirs du théâtre de Nantes. Georges Firmin-Didot, op. cit., p. 164 (bull. 30 nov. 1814).

70. Georges Firmin-Didot, op. cit., p. 102, 175-176 (Bull. des 18 août et 7 déc. 1814); Eugène Welvert, op. cit., p. 297 (bull. du 2 déc.1814); bull. du 24 août (Arch. nat. F7 3733) et du 24 août (Arch. nat. $\mathrm{F}^{7}$ 3836).

71. La rumeur de fausses pièces frappées à Elbe préoccupe la police en août et septembre 1814 (Arch. nat. $\mathrm{F}^{7}$ 3733) 
juillet 1814, on pouvait lire sur un placard affiché à la porte d'une église de Saint-Étienne : «Vive le roi pour trois jours, Bonaparte pour toujours ${ }^{72}$ ». Par conséquent l'annonce du prochain retour de l'empereur n'impliquait pas nécessairement la croyance dans la possibilité d'un accomplissement de ce qui pourrait au premier abord s'apparenter à une prédiction. Il est souvent difficile de démêler, sur la base de la lecture des rapports de police, ce qui relevait de la croyance de ce qui ressortissait à l'expression d'un vœu, d'un souhait, d'une aspiration, dont on avait peut-être conscience qu'elle n'était pas réalisable.

Autre hypothèse, celle de la rumeur-pronostic. Le bulletin du 3 septembre 1815 en donne une bonne illustration : "Quelques ouvriers qui ont l'usage de se réunir le matin sur la place de grève ont dit que l'armée de la Loire était nombreuse et en bon état, et que dans trois mois elle ferait proclamer Napoléon ${ }^{73}$.» Autrement dit on supputait, en considérant les atouts dont bénéficiait Napoléon, quelles pouvaient être ses chances de reprendre le pouvoir. Entre la fin du mois de juillet et la mi-septembre 1814, il fut beaucoup question de l'imminence d'une guerre contre l'Autriche ${ }^{74}$. Cette rumeur réveilla les espérances des "bonapartistes ${ }^{75}$, qui rapportaient que Napoléon avait pris la tête des troupes autrichiennes, ou que son beau-père, une fois acquise la victoire de ses armées, le replacerait sur le trône de France.

Plus fréquemment peut-être, la rumeur consistait en un énoncé sobre, et sans commentaire, d'un fait annonciateur d'une prochaine restauration impériale : évasion ou enlèvement de Bonaparte, révélation de sa présence en un lieu quelconque du continent européen ${ }^{76} \ldots$ Ou alors on affirmait qu'il se dirigeait vers la France ${ }^{77}$. La rumeur, dans ce cas particulier n'était pas, au sens strict, prédictive. Elle notifiait un événement qui avait déjà eu lieu, ou était en train de s'accomplir. Mais ce qui était révélé importait moins que ce qui était suggéré, ou sous-entendu : c'est-à-dire l'imminence d'une restauration impériale. Très rares, d'ailleurs, étaient les rumeurs évoquant un Napoléon ayant déjà repris les rênes du pouvoir : l'événement était inscrit dans un horizon sans cesse fuyant. Comme si la rumeur exprimait d'abord une aspiration.

72. La formule est signalée à plusieurs reprises. Bull. des 2 et 11 juill. 1814 (Arch. nat. F7 3733).

73. Une conversation de cabaret aurait été à l'origine de cette information. Bull. du 3 septembre 1815, Arch. nat. F73735.

74. Il n'est guère de semaine où la police ne fasse mention de cette rumeur. Elle est signalée fin mai, ne prend consistance qu'après le 13 juillet. Il en est encore question en octobre et en novembre. Voir l'ensemble des cartons $\mathrm{F}^{7}$ 3836, 3837, 3733 ainsi que les bulletins publiés dans Eugène Welvert, op. cit et Georges Firmin-Didot, op. cit.

75. L’expression était parfois utilisée.

76. La rumeur était associée à tout un imaginaire géographique, dont il faudrait se demander ce qu'il évoquait pour les locuteurs.

77. On disait le 26 juillet 1814 , dans des groupes de soldats réformés ou congédiés réunis quai de Gesvres : Bonaparte a quitté l'île d'Elbe pour l'Autriche. Le bulletin ajoutait : "Ces fables passent des groupes dans le bas peuple. Elles commencent à s'accréditer». Eugène Welvert, op. cit., p. 92 (bull. du 27 juill. 1814). 
L'anticipation était parfois explicite, à défaut d'être toujours précise. On annonçait un "changement prochain». Mais on entendait surtout répéter : Napoléon "va revenir", il "reviendra bientôt", il sera à Paris avant Noël ${ }^{78}$. L'information pouvait être délivrée au moyen d'une formule imagée, dont chacun saisissait parfaitement la signification. On disait alors qu'au printemps les violettes allaient refleurir (ou que les lys allaient se fermer).

La police, enfin, signala quelques rumeurs prophétiques. Dans ce cas particulier, le pronostic était délivré par une personne - en général de condition modeste, et souvent de sexe féminin ${ }^{79}$ - supposée posséder un pouvoir de divination. Une note du 25 juillet 1814 indiquait ainsi : «Il y a à Thiel près de Choisy-le-Roi [...] une femme ou fille nommée Marie-Christine Caillet qui se dit inspirée. Elle a vu Dieu pendant plus de deux mois. Elle connaît le passé et pourquoi il a eu lieu. Elle connait le présent et l'avenir, et pourquoi. » Elle sollicitait en outre une audience du ministre de l'Intérieur ${ }^{80}$. Dans la Meuse c'est une bergère qui prophétisait le retour de Napoléon ${ }^{81}$. Dans un village du Rhône, un homme, se disant protégé de Dieu, prétendait avoir lu une prophétie annonçant le retour de Napoléon ${ }^{82}$. On signalait enfin dans l'Isère des sorciers et des tireuses de carte ${ }^{83}$. La rumeur du retour de Napoléon comporta donc une dimension clairement prophétique. Mais il s'agissait d'une prophétie essentiellement orale, séculière (quand bien même le culte de Napoléon comportait une dose de religieux), et politiquement avancée. Qui s'opposait par conséquent point par point aux prophéties contre-révolutionnaires, lesquelles étaient plutôt écrites et d'inspiration catholique ${ }^{84}$.

$*$

On pourrait être tenté, au moment de conclure, d'associer cette omniprésence, dans l'espace public, de pronostics à faible degré de rationalité, à des formes de perception de l'avenir caractéristiques d'un certain stade du développement historique. Bourdieu observait que la représentation du futur comme "champ de possibles qu'il appartient au calcul d'explorer et de mâ̂triser ${ }^{85}$ était peu familière aux économies précapitalistes. La généralisation

78. Au mois de juillet 1814, des officiers assemblés déclaraient comme «certain" le retour de Bonaparte avant deux mois. Eugène Welvert, op. cit., p. 72 (bull. du 19 juill. 1814).

79. Voir les exemples postérieurs mentionnés dans François Ploux, De bouche à oreille, op. cit., p. $153-156$.

80. Une requête pas si ridicule quant on connaît l'histoire du prophète de Gallardon. Bull. du 25 juillet 1814, Arch. nat. $\mathrm{F}^{7} 3836$. Cf. Claude Guillet, La rumeur de Dieu. Apparitions, prophéties et miracles sous la Restauration, Paris, Imago, 1994.

81. Arch. nat., $\mathrm{BB}^{18} 954$ (décembre 1815).

82. Maire de Givors au préfet du Rhône, 22 et 29 août 1815, Arch. départ. Rhône, 4M 226. Voir Sudhir Hazareesingh, La légende de Napoléon, Paris, Tallandier, 2008, p. 93-94.

83. Bull. du 21 oct. 1815, Arch. nat. $\mathrm{F}^{7} 3735$.

84. Thomas A. Kselman, op. cit. Sur le prophétisme politique dans les milieux ultras, voir encore Paul Auriau «La légitimité incertaine (1814-1853) : retour sur les faux Louis XVII", Revue d'histoire XIX'e siècle, $\mathrm{n}^{\circ} 39,2009 / 2$, p. 115-127.

85. Pierre Bourdieu, "Pratiques économiques et dispositions temporelles», in Esquisse d'une 
de la monnaie, dont l'usage autorise une "comptabilité des espérances», a ainsi modifié les représentations du temps. Le développement de l'agriculture de marché aurait également favorisé la propension à anticiper rationnellement l'avenir ${ }^{86}$. La modification des modes de représentations du futur constitue sans doute l'une des dimensions du processus de politisation.

Pourtant les sociétés contemporaines ont également fait l'expérience de la prophétie politique. On pense ici aux tranchées de la Première guerre mondiale ${ }^{87}$ ou aux rumeurs apocalyptiques qui circulaient dans l'URSS des années 1920 et $1930^{88}$. Plutôt que l'expression d'une culture prophétique, les fausses nouvelles anticipatrices de 1814-1816 seraient alors le symptôme d'un certain "trouble de la conscience collective " ${ }^{89}$ associé à un contexte particulier ${ }^{90}$. Seraient notamment en cause, dans le cas qui nous concerne, l'anxiété régnante, un sentiment latent d'incertitude, un climat de tension politique à la limite du supportable ${ }^{91}$. Et ceux qui auraient pu canaliser cette colère diffuse, la relayer en la convertissant en un discours de revendication cohérent, semblent avoir surtout cherché à exacerber cette atmosphère de panique. L'exaspération populaire trouva à s'exprimer dans la rumeur napoléonienne. La propagande impériale avait bien préparé le terrain, en donnant une orientation quasi religieuse au culte du héros ${ }^{92}$. Partout, on se mit à rêver qu'une intervention extérieure, presque miraculeuse, allait libérer la France des Bourbons et de leurs alliés. La rumeur du retour de Napoléon fut une manière de contestation symbolique de l'ordre monarchique.

François Ploux est professeur d'histoire contemporaine à l'Université de Bretagne-Sud (Cerhio, UMR 6258)

théorie de la pratique précédé de trois études d'ethnologie kabyle, Paris, Seuil, 2000.

86. Claude Mesliand, cité dans Maurice Agulhon, Histoire de la France rurale, t. 3. De 1789 à 1914, Paris, Seuil, «Points», 1976, p. 457-458.

87. André Loez, 14-18. Les refus de la guerre. Une histoire des mutins, Paris, Gallimard, Folio, 2010, p. 100-101.

88. Lynne Viola, "The Peasant Nightmare: Visions of Apocalypse in the Soviet Countryside", Journal of Modern History, 62, December 1990, p. 747-770 et Nicolas Werth, «Rumeurs défaitistes et apocalyptiques dans l'URSS des années 1920 et 1930 ", Vingtième Siècle. Revue d'histoire, n 71 , juill.-sept. 2001, p. 25-35.

89. Marc Bloch, Réflexions d'un historien sur les fausses nouvelles de la guerre, Paris, Allia, 1999.

90. Les spécialistes anglo-saxons de la rumeur ont coutume d'associer ce type de phénomène à des situations "ambigües». La rumeur pourrait être assimilée à l'élaboration collective d'une interprétation d'un événement à la signification problématique. Cf. Tamotsu Shibutani, Improvised News. A Sociological Study of Rumors, Indianapolis, The Bobbs-Merril, 1966, 262 p.

91. Sans compter plusieurs autres facteurs qui n'ont pas été abordés dans cet article - par exemple le va-et-vient des soldats démobilisés, ou la présence d'armées d'occupation -, mais ont sans doute joué un rôle dans la propagation des bruits publics.

92. Jacques-Olivier Boudon, «Grand homme ou demi-dieu? La mise en place d'une religion napoléonienne", Romantisme. Revue du dix-neuvième siècle, n 100, 1998-2, p. 131-141. "Voici, dit-on, le mot d'ordre politico-maçonnico-militaire pour se reconnaître en se donnant la main : Croyez-vous en Jésus-Christ? On répond : Oui et en sa résurrection!» (Bull. du 6 sept. 1814, Arch. nat. $\left.\mathrm{F}^{7} 3836\right)$. 